\title{
INFRARED PROPERTIES OF RADIO-SELECTED SUBMILLIMETER GALAXIES IN THE SPITZER FIRST LOOK SURVEY VERIFICATION FIELD
}

\author{
D. T. Frayer, ${ }^{1}$ S. C. Chapman, ${ }^{2}$ L. Yan, ${ }^{1}$ L. Armus,${ }^{1}$ G. Helou, ${ }^{1}$ D. Fadda, ${ }^{1}$ R. Morganti, ${ }^{3}$ M. A. Garrett ${ }^{4}$ \\ P. Appleton, ${ }^{1}$ P. Choi, ${ }^{1}$ F. Fang, ${ }^{1}$ I. Heinrichsen, ${ }^{1}$ M. Im, ${ }_{1}^{5}$ M. Lacy, ${ }^{1}$ F. Marleau, ${ }^{1}$ \\ F. J. Masci, ${ }^{1}$ D. L. Shupe, ${ }^{1}$ B. T. Solfer, ${ }^{1}$ G. K. Squires, ${ }^{1}$ L. J. Storrie-Lombardi, ${ }^{1}$ \\ J. A. Surace, ${ }^{1}$ H. I. Teplitz, ${ }^{1}$ and G. WILsON ${ }^{1}$ \\ Received 2004 March 27; accepted 2004 June 7
}

\begin{abstract}
We report on submillimeter and infrared observations of 28 radio-selected galaxies in the Spitzer First Look Survey verification field. All of the radio-selected galaxies that show evidence for emission at $850 \mu \mathrm{m}$ with SCUBA have Spitzer counterparts at $24 \mu \mathrm{m}$, while only half of the radio-selected galaxies without $850 \mu \mathrm{m}$ emission have detectable counterparts at $24 \mu \mathrm{m}$. The data show a wide range of infrared colors $\left(S_{70 \mu \mathrm{m}} / S_{24 \mu \mathrm{m}}<5-30\right.$, $\left.S_{8 \mu \mathrm{m}} / S_{3.6 \mu \mathrm{m}}<0.3-4\right)$, indicative of a mixture of infrared-warm AGN-dominated and cooler starburst-dominated sources. The galaxies showing $850 \mu \mathrm{m}$ emission have Spitzer flux densities and flux density ratios consistent with the range of values expected for high-redshift $(z=1-4)$ ultraluminous infrared galaxies.
\end{abstract}

Subject headings: galaxies: active — galaxies: evolution — galaxies: formation — galaxies: starburst — infrared: galaxies

\section{INTRODUCTION}

The Spitzer Space Telescope provides us with the exciting opportunity to study the high-redshift universe at mid- and farinfrared wavelengths. The IRAS mission first uncovered the presence of infrared luminous galaxies in the local universe (Neugebauer et al. 1984), and the submillimeter/millimeter surveys with SCUBA and MAMBO have highlighted the importance of ultraluminous infrared galaxies (ULIRGs; $>10^{12} L_{\odot}$ ) at high redshift (e.g., Smail et al. 1997; Hughes et al. 1998; Bertoldi et al. 2000). Studies of high-redshift ULIRGs are important for our general understanding of galaxy evolution, since they are responsible for a significant fraction of the total energy generated by all galaxies over the history of the universe (e.g., Blain et al. 2002).

The recent spectroscopic studies of the submillimeter galaxy (SMG) population show that the redshift distribution peaks at $z \sim 2-3$ (Chapman et al. 2003a, 2004) and that the population is composed of starbursts, active galactic nuclei (AGNs), and composite AGN+starburst systems (Ivison et al. 1998, 2000; Frayer et al. 2003; Knudsen et al. 2003). Even though many SMGs show the presence of AGNs, the molecular CO line emission (Frayer et al. 1998, 1999; Neri et al. 2003) and X-ray data (Alexander et al. 2003) are consistent with the majority of the infrared emission from a population arising from star formation. The Multiband Imaging Photometer for Spitzer (MIPS; Rieke et al. 2004) allows us to directly measure the infrared colors and constrain the fraction of infrared-warm AGN-dominated versus infrared-cool starburstdominated SMGs.

\footnotetext{
${ }^{1}$ Spitzer Science Center, California Institute of Technology, MC 220-06, Pasadena, CA 91125.

${ }^{2}$ California Institute of Technology, MC 320-47, Pasadena, CA 91125.

3 ASTRON, P.O. Box 2, 7990 AA Dwingeloo, Netherlands.

4 JIVE, P.O. Box 2, 7990 AA Dwingeloo, Netherlands.

5 Astronomy Program, SEES, Seoul National University, Seoul, Korea.
}

\section{OBSERVATIONS}

Before the launch of Spitzer, we identified potential SMGs in the First Look Survey (FLS) verification field by selecting radio sources with faint optical counterparts, following previous successful selection techniques (e.g., Cowie et al. 2002; Chapman et al. 2003b). We used deep Westerbork $1.4 \mathrm{GHz}$ radio data (rms $=9 \mu \mathrm{Jy}$; Morganti et al. 2004) and deep optical NOAO $R$-band data ( $3 \sigma=26.4$ mag; Fadda et al. 2004) to derive a list of candidate sources for follow-up observations with SCUBA. In the spring of 2003, we observed 28 galaxies at the James Clerk Maxwell Telescope (JCMT) using the SCUBA two-bolometer photometry mode, achieving $\mathrm{rms}$ levels of $2-3 \mathrm{mJy}$ at $850 \mu \mathrm{m}$.

The Spitzer observations were taken as part of the extragalactic component of the FLS. ${ }^{6}$ The 28 galaxies in the sample are located within the $0.25 \mathrm{deg}^{2}$ of the FLS verification field and were observed with both the Infrared Array Camera (IRAC; Fazio et al. 2004) and MIPS. The data presented here have effective integration times of $480,336,168$, and $34 \mathrm{~s}$ for the IRAC bands and the MIPS 24, 70, and $160 \mu \mathrm{m}$ arrays, respectively. The data were reduced using the standard Spitzer Science Center (SSC) pipeline and were co-added and corrected offline as needed. The details of the data reduction will be described in future FLS data papers (IRAC: M. Lacy et al. 2004. in preparation; MIPS $24 \mu \mathrm{m}$ : D. Fadda et al. 2004, in preparation; MIPS 70 and $160 \mu$ m: D.T. Frayer et al. 2004, in preparation).

\section{RESULTS AND DISCUSSION}

\subsection{Source Identification}

We observed 28 Westerbork radio sources with SCUBA and detected seven SMGs at signal-to-noise ratios (S/Ns) of greater than 3. Fourteen sources were not detected at $850 \mu \mathrm{m}$, and the remaining seven sources have marginal results, showing positive signals of 1.5-3 $\sigma$ (Table 1, col. [5]). The Westerbork

\footnotetext{
${ }^{6}$ Spitzer Program ID 26, http://ssc.spitzer.caltech.edu/fls/extragal/spitzer.html.
} 
TABLE 1

First Look Survey Verification Field Radio-selected Candidate Submillimeter Galaxies

\begin{tabular}{|c|c|c|c|c|c|c|c|c|c|c|c|c|c|}
\hline $\begin{array}{l}\text { Source } \\
\text { (1) }\end{array}$ & $\begin{array}{c}\alpha(\mathrm{J} 2000.0) \\
(2)\end{array}$ & $\begin{array}{c}\delta(\mathrm{J} 2000.0) \\
(3)\end{array}$ & $\begin{array}{c}S_{1.4 \mathrm{GHz}} \\
(\mu \mathrm{Jy}) \\
(4)\end{array}$ & $\begin{array}{l}S_{850 \mu \mathrm{m}} \\
(\mathrm{mJy}) \\
(5)\end{array}$ & $\begin{array}{c}S_{24 \mu \mathrm{m}} \\
(\mu \mathrm{Jy}) \\
(6)\end{array}$ & $\begin{array}{l}R \text { Band } \\
\text { (mag) } \\
\text { (7) }\end{array}$ & $\begin{array}{c}S_{3.6 \mu \mathrm{m}} \\
(\mu \mathrm{Jy}) \\
(8)\end{array}$ & $\begin{array}{c}S_{4.5 \mu \mathrm{m}} \\
(\mu \mathrm{Jy}) \\
(9)\end{array}$ & $\begin{array}{c}S_{5.8 \mu \mathrm{m}} \\
(\mu \mathrm{Jy}) \\
(10)\end{array}$ & $\begin{array}{c}S_{8.0 \mu \mathrm{m}} \\
(\mu \mathrm{Jy}) \\
(11)\end{array}$ & $\begin{array}{l}\text { Submillimeter } \\
\text { (12) }\end{array}$ & $\begin{array}{c}\text { IRAC } \\
\left(1+z_{\text {phot }}\right) \\
(13)\end{array}$ & $\begin{array}{l}\text { Notes } \\
(14)\end{array}$ \\
\hline $1 \ldots \ldots \ldots \ldots$ & 171812.9 & +593922 & $750 \pm 120$ & $7.0 \pm 2.3$ & $2750 \pm 280$ & $19.68 \pm 0.05$ & $559 \pm 56$ & $365 \pm 37$ & $362 \pm 36$ & $293 \pm 29$ & $2.3 \pm 0.8$ & $<2.5$ & $\mathrm{a}$ \\
\hline $44 \ldots \ldots \ldots .$. & 171729.7 & +595429 & $74 \pm 9$ & $9.9 \pm 2.9$ & $399 \pm 40$ & $23.44 \pm 0.10$ & $\ldots$ & $\ldots$ & $\ldots$ & $\ldots$ & $4.4 \pm 0.8$ & $\ldots$ & $\mathrm{b}$ \\
\hline $48 \ldots \ldots \ldots .$. & 171733.7 & +595356 & $54 \pm 9$ & $13.1 \pm 2.9$ & $232 \pm 28$ & $23.83 \pm 0.11$ & $16.5 \pm 1.7$ & $18.0 \pm 1.8$ & $14.9 \pm 3.3$ & $<10$ & $5.0 \pm 0.8$ & $3 \pm 0.8$ & \\
\hline $115 \ldots \ldots \ldots$ & 171743.4 & +594803 & $262 \pm 26$ & $5.4 \pm 1.6$ & $373 \pm 37$ & $24.72 \pm 0.20$ & $13.0 \pm 1.3$ & $13.0 \pm 1.3$ & $<10.0$ & $28.1 \pm 3.3$ & $2.8 \pm 0.8$ & $\ldots$ & \\
\hline $119 \ldots \ldots \ldots$ & $1717 \quad 12.7$ & +594753 & $81 \pm 9$ & $10.2 \pm 2.8$ & $146 \pm 28$ & $>26.4$ & $43.3 \pm 4.3$ & $44.0 \pm 4.4$ & $42.9 \pm 4.3$ & $25.5 \pm 3.3$ & $4.3 \pm 0.8$ & $3 \pm 0.8$ & $\mathrm{c}$ \\
\hline $199 \ldots \ldots \ldots$ & 171729.4 & +594113 & $116 \pm 12$ & $7.5 \pm 2.5$ & $301 \pm 30$ & $23.44 \pm 0.14$ & $35.4 \pm 3.5$ & $39.9 \pm 4.0$ & $49.3 \pm 4.9$ & $48.6 \pm 4.9$ & $3.7 \pm 0.8$ & $\ldots$ & $z=1.06, \mathrm{~d}$ \\
\hline $208 \ldots \ldots \ldots$ & 171810.9 & +594041 & $77 \pm 9$ & $6.8 \pm 1.9$ & $78 \pm 28$ & $22.62 \pm 0.10$ & $24.5 \pm 2.5$ & $24.0 \pm 2.4$ & $<10$ & $19.3 \pm 3.3$ & $3.9 \pm 0.8$ & $\ldots$ & \\
\hline $45 \mathrm{~A} \ldots \ldots \ldots$ & 171747.5 & +595424 & $80 \pm 9$ & $7.2 \pm 2.5$ & $200 \pm 28$ & $24.12 \pm 0.14$ & $26.0 \pm 2.6$ & $27.4 \pm 2.7$ & $36.5 \pm 3.7$ & $47.4 \pm 4.7$ & $4.0 \pm 0.8$ & & $\mathrm{e}$ \\
\hline $47 \ldots \ldots \ldots$ & 171722.5 & +595412 & $100 \pm 10$ & $4.9 \pm 2.4$ & $498 \pm 50$ & $25.31 \pm 0.22$ & $28.2 \pm 2.8$ & $45.6 \pm 4.6$ & $68.1 \pm 6.8$ & $111.6 \pm 11.2$ & $3.4 \pm 0.8$ & & $\mathrm{c}, \mathrm{f}$ \\
\hline $73 \ldots \ldots \ldots$ & 171757.9 & +595200 & $118 \pm 12$ & $5.8 \pm 2.1$ & $135 \pm 28$ & $>26.4$ & $29.4 \pm 2.9$ & $35.5 \pm 3.6$ & $30.7 \pm 3.3$ & $27.2 \pm 3.3$ & $3.4 \pm 0.8$ & $3 \pm 0.8$ & $\mathrm{c}$ \\
\hline $139 \ldots \ldots \ldots$ & 171823.2 & +594553 & $215 \pm 22$ & $5.0 \pm 2.8$ & $444 \pm 44$ & $22.62 \pm 0.05$ & $36.9 \pm 3.7$ & $32.5 \pm 3.3$ & $27.9 \pm 3.3$ & $24.1 \pm 3.3$ & $2.9 \pm 0.8$ & $<2.5$ & \\
\hline $145 \ldots \ldots \ldots$ & 171746.2 & +594517 & $66 \pm 9$ & $8.7 \pm 4.0$ & $161 \pm 28$ & $>26.4$ & $7.2 \pm 1.0$ & $7.7 \pm 1.0$ & $<10$ & $19.1 \pm 3.3$ & $4.3 \pm 0.8$ & $\ldots$ & $\mathrm{c}$ \\
\hline $156 \ldots \ldots \ldots$ & 171816.8 & +594430 & $82 \pm 9$ & $4.4 \pm 2.5$ & $368 \pm 37$ & $25.64 \pm 0.37$ & $9.7 \pm 1.0$ & $12.9 \pm 1.3$ & $25.0 \pm 3.3$ & $21.0 \pm 3.3$ & $3.5 \pm 0.8$ & $3.5 \pm 0.8$ & \\
\hline $191 \ldots \ldots \ldots$. & 171715.5 & +594202 & $145 \pm 15$ & $3.8 \pm 2.5$ & $277 \pm 28$ & $25.35 \pm 0.34$ & $<3$ & $<3$ & $<10$ & $<10$ & $3.0 \pm 0.8$ & $\ldots$ & \\
\hline $75 \ldots \ldots \ldots . .$. & 171801.7 & +595147 & $38 \pm 9$ & $<7.5$ & $416 \pm 42$ & $>26.4$ & $20.0 \pm 2.0$ & $24.6 \pm 2.5$ & $27.2 \pm 3.3$ & $19.8 \pm 3.3$ & $<4.8$ & $3.5 \pm 0.8$ & $\mathrm{c}$ \\
\hline 79A.......... & 171722.8 & +595130 & $114 \pm 11$ & $<6.6$ & $408 \pm 41$ & $23.30 \pm 0.17$ & $54.2 \pm 5.4$ & $38.9 \pm 3.9$ & $36.6 \pm 3.7$ & $25.7 \pm 3.3$ & $<3.6$ & $<2.5$ & $\mathrm{~g}$ \\
\hline $85 \ldots \ldots \ldots \ldots$ & 171812.3 & +595056 & $44 \pm 9$ & $<8.4$ & $<84$ & $21.93 \pm 0.05$ & $40.1 \pm 4.0$ & $26.4 \pm 2.6$ & $23.9 \pm 3.3$ & $16.5 \pm 3.3$ & $<4.7$ & $<2.5$ & \\
\hline $91 \ldots \ldots \ldots$. & 171743.7 & +595022 & $43 \pm 9$ & $<7.8$ & $331 \pm 33$ & $>26.4$ & $13.3 \pm 1.3$ & $17.9 \pm 1.8$ & $15.5 \pm 3.3$ & $18.3 \pm 3.3$ & $<4.7$ & $\ldots$ & $\mathrm{c}$ \\
\hline $99 \ldots \ldots \ldots . . .$. & 171706.4 & +594925 & $139 \pm 14$ & $<8.4$ & $<84$ & $23.50 \pm 0.15$ & $36.3 \pm 3.6$ & $25.3 \pm 2.5$ & $10.0 \pm 3.3$ & $<10$ & $<3.6$ & $<2.5$ & $\mathrm{~h}$ \\
\hline $108 \ldots \ldots \ldots$ & 171741.2 & +594836 & $1080 \pm 130$ & $<8.1$ & $<84$ & $>26.4$ & $<3$ & $<3$ & $<10$ & $<10$ & $<2.2$ & $\ldots$ & \\
\hline $109 \ldots \ldots \ldots$ & 171738.5 & +594832 & $765 \pm 110$ & $<6.6$ & $<84$ & $24.19 \pm 0.14$ & $<3$ & $<3$ & $<10$ & $<10$ & $<2.2$ & $\ldots$ & \\
\hline $128 \mathrm{~B} \ldots \ldots$ & 171747.0 & +594712 & $62 \pm 9$ & $<9.0$ & $<84$ & $25.41 \pm 0.26$ & $4.1 \pm 1.0$ & $3.6 \pm 1.0$ & $<10$ & $<10$ & $<4.4$ & $\ldots$ & $\mathrm{i}$ \\
\hline $155 \ldots \ldots \ldots$ & 171756.0 & +594432 & $60 \pm 9$ & $<7.2$ & $<84$ & $24.60 \pm 0.27$ & $30.0 \pm 3$ & $32.7 \pm 3.3$ & $21.7 \pm 3.3$ & $<10$ & $<4.3$ & $3 \pm 0.8$ & \\
\hline $136 \ldots \ldots \ldots$ & 171711.6 & +594621 & $108 \pm 9$ & $<6.9$ & $292 \pm 29$ & $23.89 \pm 0.16$ & $21.8 \pm 2.2$ & $23.5 \pm 2.4$ & $19.0 \pm 3.3$ & $17.3 \pm 3.3$ & $<3.7$ & $3 \pm 0.8$ & \\
\hline $146 \ldots \ldots \ldots$ & 171812.9 & +594454 & $627 \pm 63$ & $<9.9$ & $217 \pm 28$ & $23.83 \pm 0.10$ & $39.3 \pm 3.9$ & $27.4 \pm 2.7$ & $15.7 \pm 3.3$ & $<10$ & $<2.6$ & $<2.5$ & \\
\hline $150 \ldots \ldots \ldots$ & 171742.4 & +594456 & $68 \pm 9$ & $<6.9$ & $259 \pm 28$ & $23.37 \pm 0.14$ & $18.7 \pm 1.9$ & $11.8 \pm 1.2$ & $<10$ & $21.8 \pm 3.3$ & $<4.1$ & $\ldots$ & $z=0.84, \mathrm{~d}$ \\
\hline $198 \ldots \ldots \ldots$. & 171721.4 & +594113 & $114 \pm 12$ & $<8.1$ & $<84$ & $>26.4$ & $21.2 \pm 2.1$ & $22.0 \pm 2.2$ & $13.3 \pm 3.3$ & $13.8 \pm 3.3$ & $<3.7$ & $<2.5$ & $\mathrm{c}$ \\
\hline $211 \ldots \ldots \ldots$ & 171821.3 & +594027 & $356 \pm 36$ & $<8.4$ & $432 \pm 43$ & $26.16 \pm 0.42$ & $32.3 \pm 3.2$ & $41.7 \pm 4.2$ & $60.1 \pm 6.0$ & $89.2 \pm 8.9$ & $<2.9$ & $\ldots$ & $\mathrm{c}$ \\
\hline
\end{tabular}

Notes.-Col. (1): Radio source identifier. Cols. (2)-(3): Radio positions that are accurate to about $\pm 2^{\prime \prime}$. Units of right ascension are hours, minutes, and seconds, and units of declination are degrees, arcminutes, and arcseconds. Col. (4): Radio flux densities from the Westerbork and VLA data. Col. (5): SCUBA $850 \mu \mathrm{m}$ measurements. Sources $1-208$ have greater than $3 \sigma 850 \mu \mathrm{m}$ detections, sources $45 \mathrm{~A}-191$ have 1.5-3 $\sigma 850 \mu \mathrm{m}$ measurements, and sources 75-211 are nondetections at $850 \mu \mathrm{m}$. All flux density and magnitude limits are given as $3 \sigma$. Col. (6): MIPS $24 \mu \mathrm{m}$ measurements. Col. (7): Optical $R$-band magnitudes from NOAO $4 \mathrm{~m}$. Cols. (8)-(11): IRAC flux densities. Col. (12): Photometric redshift estimate of $(1+z)\left(T_{d} / 40 \mathrm{~K}\right)^{-1}$ based on the submillimeter to radio relationship of Carilli \& Yun (1999), accounting for the degeneracy of redshift and dust temperature in the relationship (Blain 1999). Col. (13): Photometric redshift based on the assumed rest-frame 1.6 $\mu \mathrm{m}$ peak in the IRAC SED. Col. (14): Notes as follows. (a) Measurements summed over all five components (Fig. 1). Source 1 is also detected at longer wavelengths with $S_{70} \mu \mathrm{m}=30 \pm 6 \mathrm{mJy}$ and $S_{160 \mu \mathrm{m}}=130 \pm 26 \mathrm{mJy}$. Sources not detected in the 70 and $160 \mu \mathrm{m}$ MIPS bands have $3 \sigma$ limits of $\lesssim 10$ and $\lesssim 50 \mathrm{mJy}$, respectively. (b) Detected in IRAC bands, but IRAC measurements are confused with nearby bright optical source. (c) ERO based on high $S_{3.6} \mu \mathrm{m} / S_{R}$ flux density ratio. (d) Spectroscopic redshift based on Keck DEIMOS data (P. Choi et al. 2004, in preparation). (e) Brighter radio component 45A has a $24 \mu \mathrm{m}$ counterpart and is adopted as the primary counterpart, since $45 \mathrm{~B}$ has no associated $24 \mu \mathrm{m}$ source. (f) Detected in MIPS $70 \mu \mathrm{m}$ with $S_{70} \mu \mathrm{m}=13 \pm 3 \mathrm{mJy}$. (g) Brighter radio component $79 \mathrm{~A}$ has a $24 \mu \mathrm{m}$ counterpart and "merger-like" morphology with two optical components. (h) Source 99 has two optical components showing a merger-like morphology. (i) Neither radio component of source 128 has a $24 \mu \mathrm{m}$ counterpart; $128 \mathrm{~B}$ is adopted as the counterpart for flux density comparisons since there are no optical or IRAC counterparts associated with 128A. 
radio data have a resolution of $14^{\prime \prime} \times 11^{\prime \prime}$, which is well matched to the SCUBA data but is not sufficient to obtain reliable counterparts in general for the optical and Spitzer data. We used the higher resolution (5"0) Very Large Array (VLA; $1.4 \mathrm{GHz}$ ) data of the field (Condon et al. 2003) to obtain more accurate radio positions. The resolution of the VLA data is well matched to the MIPS $24 \mu \mathrm{m}$ resolution of $6^{\prime \prime}$. Although the VLA data have lower $\mathrm{S} / \mathrm{Ns}(\mathrm{rms} \sim 20 \mu \mathrm{Jy})$, the Westerbork sources were typically detected at $\gtrsim 3 \sigma$ in the VLA image, providing radio positional errors of about $1^{\prime \prime}-2^{\prime \prime}$. The offsets between radio and MIPS $24 \mu \mathrm{m}$ positions are typically less than $2^{\prime \prime}$, consistent within the positional uncertainties of the radio data and the 1".5$2^{\prime \prime}$ positional uncertainties of the MIPS $24 \mu \mathrm{m}$ data set. Based on the $24 \mu \mathrm{m}$ counts in the FLS field (Marleau et al. 2004), the probability of a chance coincidence within $2^{\prime \prime}$ is about $1 \%$ for $24 \mu \mathrm{m}$ sources brighter than $100 \mu \mathrm{Jy}$. Hence, confusion at $24 \mu \mathrm{m}$ is not a significant issue for this study. After the identification of the MIPS $24 \mu \mathrm{m}$ and $R$-band counterparts based on the radio positions, the corresponding MIPS $24 \mu \mathrm{m}$ and optical positions were used to identify the appropriate IRAC counterparts.

Four of the Westerbork sources have multiple radio components at the resolution of the VLA data $(1,45,79$, and 128). Source 1 is the brightest Spitzer source and is composed of five components, all detected in the MIPS $24 \mu \mathrm{m}, R$, and IRAC bands. The details of the source identification are provided in Table 1. Figure 1(Plate 1) shows the MIPS $24 \mu \mathrm{m}$ and IRAC $3.6 \mu \mathrm{m}$ images for the seven SMGs detected at $850 \mu \mathrm{m}$ with $\mathrm{S} / \mathrm{N}>3$.

All 14 sources showing $850 \mu \mathrm{m}$ emission above the $1.5 \sigma$ level have $24 \mu \mathrm{m}$ counterparts, although source 208 is only detected at about the $2.5-3 \sigma$ level at $24 \mu \mathrm{m}$. The detection of $24 \mu \mathrm{m}$ counterparts associated even with the marginal $850 \mu \mathrm{m}$ sources gives credence to the reliability of the greater than $3 \sigma$ SMG sources. In comparison with the 14 radio sources without SCUBA detections, only half have $24 \mu \mathrm{m}$ counterparts. The detection of $24 \mu \mathrm{m}$ counterparts for this sample of radioselected SMGs is consistent with the high fraction of $24 \mu \mathrm{m}$ counterparts found for the MAMBO and SCUBA sources in the Lockman Hole (Ivison et al. 2004; Egami et al. 2004).

Seven sources in the full sample do not have optical counterparts down to $R=26.4 \mathrm{mag}$, and only three are not detected by IRAC. The only source without any optical or IRAC counterpart is source 108, which is the brightest radio galaxy in the sample (likely a radio-loud AGN). Two sources (199 [z=1.06] and $150[z=0.84])$ in the sample have spectroscopic redshifts from the ongoing Keck DEIMOS redshift survey (P. Choi et al. 2004, in preparation). The low redshift of $z=1.06$ for the 850 $\mu \mathrm{m}$ source 199 implies a cool dust temperature of $20 \mathrm{~K}$, assuming a temperature-dependent submillimeter to radio redshift relationship (Blain 1999).

\subsection{Infrared Properties}

The MIPS $24 \mu \mathrm{m}$ flux densities for the galaxies detected at $850 \mu \mathrm{m}$ are consistent with expectations, assuming standard ULIRG spectral energy distributions (SEDs) at the typical redshifts of $z \sim 2-3$ found for the SMG population (Chapman et al. 2003a, 2004). Figure 2 shows a range of $S_{24 \mu \mathrm{m}} / S_{1.4 \mathrm{GHz}}$ flux density ratios for the population that may reflect a wide distribution of infrared colors. All of the galaxies showing $850 \mu \mathrm{m}$ emission lie within the range of values expected for local ULIRGs redshifted to $z=1-4$. Galaxies without $850 \mu \mathrm{m}$ emission show a wider range of $S_{24 \mu \mathrm{m}} / S_{1.4 \mathrm{GHz}}$ flux density ratios. About half of the radio sources without detectable $850 \mu \mathrm{m}$ emission have properties consistent with ULIRGs,

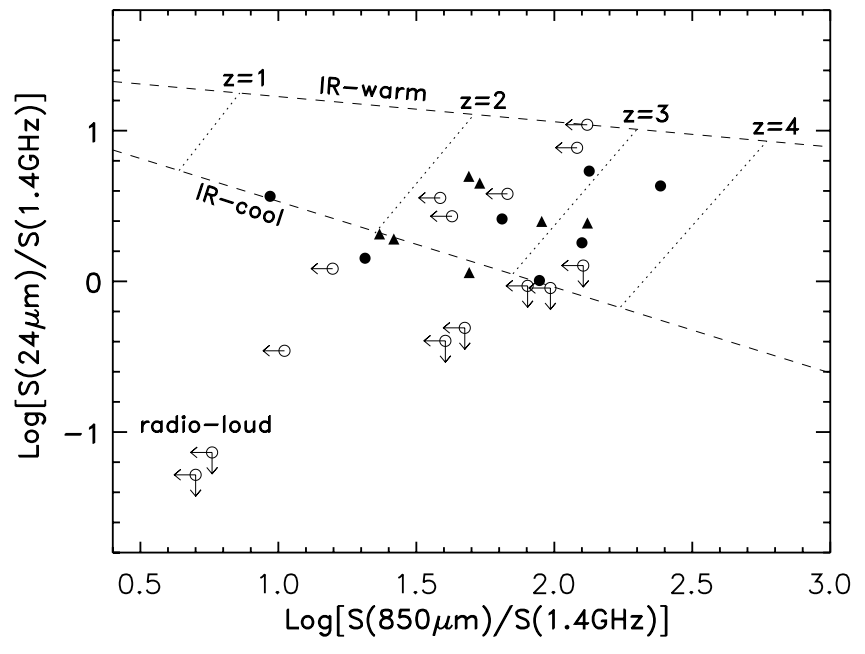

FIG. 2.-The $S_{24 \mu \mathrm{m}} / S_{1.4 \mathrm{GHz}}$ flux density ratios as a function of the $S_{850 \mu \mathrm{m}} /$ $S_{1.4 \mathrm{GHz}}$ flux density ratio. The filled circles are the greater than $3 \sigma \mathrm{SMGs}$, the open circles are the radio sources without $850 \mu \mathrm{m}$ emission, and the filled triangles are the sources with $850 \mu \mathrm{m}$ measurements of $1.5-3 \sigma$. All limits are $2 \sigma$. The dashed lines show the expected ratios assuming power-law approximations for the infrared, submillimeter, and radio emission. The top dashed line represents an infrared-warm ULIRG, while the bottom dashed line represents an infrared-cool ULIRG. Redshift estimates are shown by the dotted lines. The observed ratios are consistent with the SEDs of local ULIRGs at the expected redshifts of $z \sim 1-4$ for the SMG population. Strong radio galaxies that do not obey the far-infrared to radio correlation are located on the lower left.

while the other half show excess radio emission compared to their $24 \mu \mathrm{m}$ emission, consistent with different degrees of radio-loudness.

MIPS $70 \mu \mathrm{m}$ observations can constrain the infrared colors of the SMGs. Infrared-warm, AGN-dominated sources are expected to have flux density ratios of $S_{70 \mu \mathrm{m}} / S_{24 \mu \mathrm{m}} \sim 5$, while starburst-dominated, infrared-cool sources are expected to have ratios of $S_{70 \mu \mathrm{m}} / S_{24 \mu \mathrm{m}} \sim 10$. We only detect two sources at $70 \mu \mathrm{m}$, both of which are cool in the infrared (source 1, $S_{70 \mu \mathrm{m}} / S_{24 \mu \mathrm{m}}=11$; source $47, S_{70 \mu \mathrm{m}} / S_{24 \mu \mathrm{m}}=26$ ). To derive an estimated average $S_{70 \mu \mathrm{m}} / S_{24 \mu \mathrm{m}}$ ratio for the SMG population, we co-added the $70 \mu \mathrm{m}$ data at the radio positions of the sources showing $850 \mu \mathrm{m}$ emission. We find an upper limit of $S_{70 \mu \mathrm{m}}<1.2 \mathrm{mJy}(2 \sigma)$. This corresponds to an infrared color of $S_{70 \mu \mathrm{m}} / S_{24 \mu \mathrm{m}}<5$, leaving out the two sources with $70 \mu \mathrm{m}$ detections. Including the two $70 \mu \mathrm{m}$ detections, the average ratio for the SMG population is $S_{70 \mu \mathrm{m}} / S_{24 \mu \mathrm{m}}<7$, which is slightly lower than expected if the population is dominated by star formation. Taken at face value, these results could suggest that many SMGs are infrared-warm AGNs, contrary to previous conclusions that the population is dominated by star formation. However, the lower than expected $S_{70 \mu \mathrm{m}} / S_{24} \mu \mathrm{m}$ ratios may arise from strong $7.7 \mu \mathrm{m}$ PAH emission redshifted into the $24 \mu \mathrm{m}$ band, given that the median redshift for the SMG population is $z \sim 2.4$ (Chapman et al. 2003a, 2004).

The SEDs in the IRAC bands can be used to estimate redshifts from the rest-frame $1.6 \mu \mathrm{m}$ peak expected for starforming systems (e.g., Egami et al. 2004) and to help identify AGNs showing hot dust implied by their red IRAC colors (e.g., Ivison et al. 2004). For sources showing a significant bump in the IRAC bands, the implied redshifts are generally consistent (Table 1, cols. [12] and [13]) with the redshift estimates derived from the submillimeter to radio spectral index (Carilli \& Yun 1999; Blain 1999). The discrepant photometric redshifts for sources 48 and 119 may indicate cool dust temperatures $\left(T_{d}<40 \mathrm{~K}\right)$ for these SMGs. 


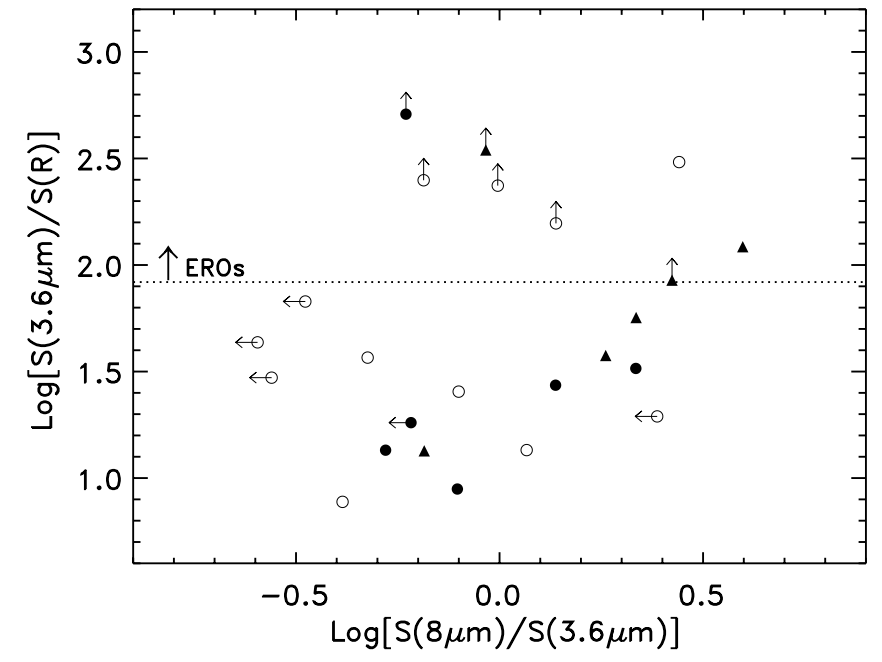

FIG. 3.-The $S_{3.6 \mu \mathrm{m}} / S_{R}$ flux density ratios as a function of IRAC color given by the $S_{8 \mu \mathrm{m}} / S_{3.6 \mu \mathrm{m}}$ flux density ratio for sources with detections at $3.6 \mu \mathrm{m}$. Symbols are the same as in Fig. 1. EROs are located above the dotted line, corresponding to $R-[3.6]>7.5$ (or $R-K>6$ ). The data show a wide range of IRAC colors.

Of the sources with $850 \mu \mathrm{m}$ emission and IRAC detections, only source 47 shows a strong increase in flux density as a function of wavelength across the IRAC bands consistent with an AGN. However, source 47 also has the highest $S_{70 \mu \mathrm{m}} / S_{24} \mu \mathrm{m}$ ratio in the sample $\left(S_{70 \mu \mathrm{m}} / S_{24 \mu \mathrm{m}}=26\right)$, suggesting that it is likely an infrared-cool starburst that could be at $z \gtrsim 4$ and/or highly reddened because of dust extinction. Hence, IRAC colors are not always conclusive for determining the properties at longer infrared wavelengths.

\subsection{Extremely Red Objects}

A significant fraction of SMGs is composed of extremely red objects (EROs; Smail et al. 1999; Frayer et al. 2004). The
ERO definition of $R-K>6$ (Thompson et al. 1999) corresponds to a flux density ratio of $S_{3.6 \mu \mathrm{m}} / S_{R} \geq 85$. Using this criterion, we identify eight EROs (Fig. 3). The 30\% (8/27) fraction of EROs in this sample is larger than the $8 \%$ found for the total microjansky radio population (Smail et al. 2002), presumably since the SCUBA targets in this sample were generally selected on the basis of being faint and red in the optical bands. Figure 3 shows a wide range of IRAC colors for the SMG population, and no correlation is observed between the IRAC colors and the $R-[3.6]$ colors. Another interesting result is that most of the radio-selected EROs without detectable $850 \mu \mathrm{m}$ emission (6/7) are bright at $24 \mu \mathrm{m}$, indicating that these sources are infrared-bright galaxies (likely highredshift ULIRGs) below the current SCUBA detection limits.

\section{CONCLUSIONS}

All of the radio-selected SMGs in the sample have Spitzer counterparts, showing a wide range of infrared colors consistent with ULIRGs at $z=1-4$. The combination of accurate radio positions and $24 \mu \mathrm{m}$ detections is a powerful tool for the identification of SMGs. More sensitive observations are required in the MIPS 70 and $160 \mu \mathrm{m}$ bands to measure the infrared colors of the SMG population. In addition, observations with the Spitzer IRS are needed to determine the level at which polycyclic aromatic hydrocarbon (PAH) emission contributes to the MIPS $24 \mu \mathrm{m}$ flux densities and to help determine the AGN fraction of the population. In general, Spitzerselected ULIRGs will be biased toward AGNs, except within specific redshift bins associated with the PAH features, which may be biased toward starbursts.

We thank the staff at the JCMT and the Spitzer Science Center for their support of these observations. This work is based in part on observations made with the Spitzer Space Telescope, which is operated by the Jet Propulsion Laboratory, California Institute of Technology, under NASA contract 1407.
Alexander, D. M., et al. 2003, AJ, 125, 383

Bertoldi, F., et al. 2000, A\&A, 360, 92

Blain, A. W. 1999, MNRAS, 309, 955

Blain, A. W., Smail, I., Ivison, R. J., Kneib, J.-P., \& Frayer, D. T. 2002, Phys. Rep., 369, 111

Carilli, C. L., \& Yun, M. S. 1999, ApJ, 513, L13

Chapman, S. C., Blain, A. W., Ivison, R. J., \& Smail, I. R. 2003a, Nature, 422,695

. 2004, ApJ, submitted

Chapman, S. C., et al. 2003b, ApJ, 585, 57

Condon, J. J., Cotton, W. D., Yin, Q. F., Shupe, D. L., Storrie-Lombardi, L. J., Helou, G., Soifer, B. T., \& Werner, M. W. 2003, AJ, 125, 2411

Cowie, L. L., Barger, A. J., \& Kneib, J.-P. 2002, AJ, 123, 2197

Egami, E., et al. 2004, ApJS, 154, 130

Fadda, D., Jannuzi, B., Ford, A., \& Storrie-Lombardi, L. J. 2004, AJ, 128, 1

Fazio, G. G., et al. 2004, ApJS, 154, 10

Frayer, D. T., Armus, L., Scoville, N. Z., Blain, A. W., Reddy, N. A., Ivison, R. J., \& Smail, I. 2003, AJ, 126, 73

Frayer, D. T., Ivison, R. J., Scoville, N. Z., Yun, M., Evans, A. S., Smail, I., Blain, A. W., \& Kneib, J.-P. 1998, ApJ, 506, L7

Frayer, D. T., Reddy, N. A., Armus, L., Blain, A. W., Scoville, N. Z., \& Smail, I. 2004a, AJ, 127, 728
REFERENCES

Frayer, D. T., et al. 1999, ApJ, 514, L13

Hughes, D., et al. 1998, Nature, 394, 241

Ivison, R. J., Smail, I., Barger, A. J., Kneib, J.-P., Blain, A. W., Owen, F. N., Kerr, T. H., \& Cowie, L. L. 2000, MNRAS, 315, 209

Ivison, R. J., Smail, I., Le Borgne, J.-F., Blain, A. W., Kneib, J.-P., Bézecourt, J., Kerr, T. H., \& Davies, J. K. 1998, MNRAS, 298, 583

Ivison, R. J., et al. 2004, ApJS, 154, 124

Knudsen, K. K., van der Werf, P. P., \& Jaffe, W. 2003, A\&A, 411, 343

Marleau, F., et al. 2004, ApJS, 154, 66

Morganti, R., Garrett, M.A., Chapman, S., Baan, W., Helou, G., \& Soifer, T. 2004, A\&A, in press (astro-ph/0405418)

Neri, R., et al. 2003, ApJ, 597, L113

Neugebauer, G., et al. 1984, ApJ, 278, L1

Rieke, G., et al. 2004, ApJS, 154, 25

Smail, I., Ivison, R. J., \& Blain, A. W. 1997, ApJ, 490, L5

Smail, I., Ivison, R. J., Kneib, J.-P., Cowie, L. L., Blain, A. W., Barger, A. J., Owen, F. N., \& Morrison, G. 1999, MNRAS, 308, 1061

Smail, I., Owen, F. N., Morrison, G. E., Keel, W. C., Ivison, R. J., \& Ledlow, M. J. 2002, ApJ, 581, 844

Thompson, D., et al. 1999, ApJ, 523, 100 

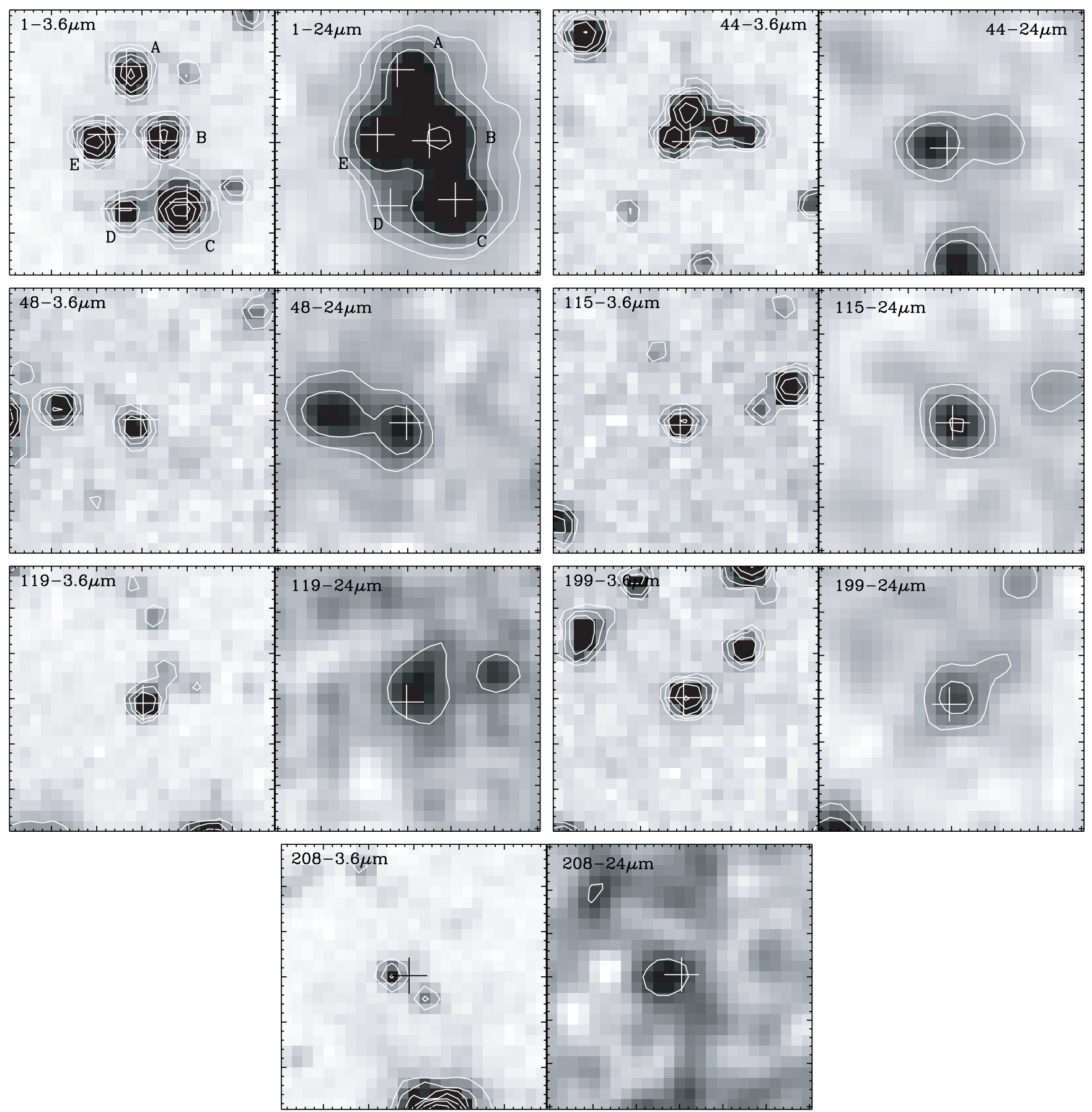

FIG. 1.-IRAC $3.6 \mu \mathrm{m}$ and MIPS $24 \mu \mathrm{m}$ images of the seven SMGs detected at $850 \mu \mathrm{m}$ with $\mathrm{S} / \mathrm{N}>3$. Each panel is approximately $30^{\prime \prime}$ in size (north is up and east is left), with tick marks every $1^{\prime \prime}$. The gray scale is plotted on a logarithmic scale, and the contours start at $3 \sigma$ and increase by factors of 2 . The crosses represent the positions of the radio counterparts. 